\title{
Analytic representations of rovibrational dipole matrix elements for the CO molecule and its isotopomers ${ }^{\star}$
}

\author{
J.M. Huré and E. Roueff \\ DAEC et URA 173 du CNRS, Observatoire de Paris-Meudon, Place Jules Janssen, F-92195 Meudon Principal, France \\ e-mails: roueff@obspm.fr and hure@mesiom.obspm.fr
}

Received July 20; accepted December 18, 1995

\begin{abstract}
We report new calculations of transition matrix elements for more than thirty thousand infrared lines of the CO molecule and its isotopomers inside the $\mathrm{X}^{1} \Sigma^{+}$ground electronic state. These lines belong to the $\Delta v=1$, 2,3 bands involving very high excited states such as $v^{\prime} \leq 41$ and $J^{\prime} \leq 133$. The transition matrix elements have been calculated from an accurate solution of the Schrödinger equation by using two different theoretical electric dipole moment functions (Werner 1981; Langhoff \& Bauschlicher 1995). For low lying levels, our data compare satisfactorily with experimental ones and related works (Goorvitch \& Chackerian 1994a, b; Chackerian et al. 1994, Goorvitch 1994). Moreover, we make predictions for oscillator strengths and Einstein coefficients involving high excited transitions. In addition, we report simple analytic formulae that fit our results for ${ }^{12} \mathrm{C}^{16} \mathrm{O}$ with an accuracy better than $10^{-2}$ as a function of $m=\left[J^{\prime}\left(J^{\prime}+1\right)-J^{\prime \prime}\left(J^{\prime \prime}+1\right)\right] / 2$ for each vibrational sequence and band. They offer an easy way to compute the various intensities of these rovibrational transitions whose transition energies may be introduced via the available Dunham coefficients.
\end{abstract}

Key words: molecular data — ISM: molecules

\section{Introduction}

The determination of the rovibrational intensities of carbon monoxide is important in our understanding of various astrophysical media such as stellar and planetary atmospheres, circumstellar envelopes and interstellar clouds. Amongst the recent attempts (Huré \& Roueff 1993; Goorvitch \& Chackerian 1994a, b; Chackerian et al. 1994; Goorvitch 1994) to get systematic results of the intensities of fundamental and overtone rovibrational bands, significant discrepancies have been found. As discussed in Chackerian et al. (1994), these differences come from different choices of the Electric Dipole Moment Function (EDMF hereafter) - the behaviour of which is very uncertain far from the equilibrium internuclear separation.

Laboratory measurements may provide the most accurate constraints on the EDMF from infrared emission line intensity ratios for low rovibrational states. However, their reliability is less obvious for intermediate and high energy levels which imply high excitation conditions and large internuclear separations. Alternatively, the accuracy of theoretical calculations for the EDMF is not limited at interatomic distances far from the equilibrium distance.

Send offprint requests to: E. Roueff

${ }^{\star}$ Tables 5 to 7 only available in electronic form at CDS via ftp 150.79.128.5
We present in this paper two new sets of infrared dipolar transition matrix elements with $v^{\prime} \leq 41$ and $J^{\prime} \leq 133$ for ${ }^{12} \mathrm{C}^{16} \mathrm{O},{ }^{13} \mathrm{C}^{16} \mathrm{O},{ }^{12} \mathrm{C}^{18} \mathrm{O}$ and ${ }^{12} \mathrm{C}^{17} \mathrm{O}$ molecules in their electronic ground state. The calculations are performed by using the two theoretical ab-initio EDMFs of Werner (1981) and Langhoff \& Bauschlicher (1995). Both these EDMFs are in very good agreement with the experimental results for low excitation transitions of the fundamental band (Goorvitch \& Chackerian 1994a, b). In addition, our transition matrix elements are fitted with fourth degree polynomial expansions which reproduce the original data with an accuracy of the order of $10^{-3}$ for most of the transitions.

Using the energy terms given by the mass independent Dunham coefficients (Farrenq et al. 1991; Authier et al. 1993), these new sets allow straightforward calculations of rovibrational intensities of the infrared transitions of $\mathrm{CO}$ and its minor isotopomers.

\section{Theoretical background}

In the Born-Oppenheimer approximation, the energy levels $E_{v, J}$ of a molecule in a ${ }^{1} \Sigma^{+}$ground state and the corresponding radial wave functions $\Psi_{v, J}(R)$ are solutions of the Schrödinger nuclear equation (see Steinfeld 1989): 


$$
\begin{aligned}
& {\left[-\frac{h^{2}}{8 \pi^{2} \mu}\left(\frac{\mathrm{d}^{2}}{\mathrm{~d} R^{2}}+\frac{J(J+1)}{R^{2}}\right)+V(R)\right]} \\
& \Psi_{v, J}(R)=E_{v, J} \Psi_{v, J}(R)
\end{aligned}
$$

Here, $\mu$ is the reduced mass of the two nuclei, $V(R)$ is the electronic potential depending on the radial distance $R$, and $v$ and $J$ denote respectively the vibrational and the rotational quantum numbers. We point out that this equation does not assume any separation between rotation and vibration motions. It remains valid as far as the BornOppenheimer approximation holds.

Table 1. EDMF and its first derivative at equilibrium distance for EDMF1 (Werner 1981), EDMF2 (Langhoff \& Bauschlicher 1995) and EDMF3 (Chackerian et al. 1984)

\begin{tabular}{lllll} 
& & EDMF1 & EDMF2 & EDMF3 \\
\hline$M\left(R_{\mathrm{e}}\right)$ & $(\mathrm{D})$ & -0.237 & -0.123 & -0.123 \\
$d M /\left.d R\right|_{R_{e}}\left(\mathrm{D} / \mathrm{a}_{0}\right)$ & +1.682 & +1.693 & +1.674 \\
\hline
\end{tabular}

Table 2. Values of the reduced masses for the different isotopes of $\mathrm{CO}$

\begin{tabular}{lc} 
Isotope & Reduced mass (amu) \\
\hline${ }^{12} \mathrm{C}^{16} \mathrm{O}$ & 6.856208709 \\
${ }^{13} \mathrm{C}^{16} \mathrm{O}$ & 7.172412452 \\
${ }^{12} \mathrm{C}^{18} \mathrm{O}$ & 7.199865602 \\
${ }^{12} \mathrm{C}^{17} \mathrm{O}$ & 7.034334287 \\
\hline
\end{tabular}

Both absorption oscillator strengths (or $f$-values) and emission Einstein $A$ coefficients depend on the energy levels and on the rovibrational matrix elements (or $D$ values). For a given transition involving a lower level $\left(v^{\prime \prime}\right.$, $\left.\mathrm{J}^{\prime \prime}\right)$ and an upper one $\left(v^{\prime}, J^{\prime}\right)$, the $f$-value and the $A$ coefficient are respectively:

$$
\begin{aligned}
& f_{v^{\prime \prime}, J^{\prime \prime}}^{v^{\prime}, J^{\prime}}=\frac{8 \pi^{2} m_{\mathrm{e}}}{3 h^{2} \mathrm{e}^{2}} \frac{E_{v^{\prime}, J^{\prime}}-E_{v^{\prime \prime}, J^{\prime \prime}}}{2 J^{\prime \prime}+1} S_{v^{\prime \prime}, J^{\prime \prime}}^{v^{\prime}, J^{\prime}}\left|D_{v^{\prime \prime}, J^{\prime \prime}}^{v^{\prime}, J^{\prime}}\right|^{2} \\
& A_{v^{\prime \prime}, J^{\prime \prime}}^{v^{\prime}, J^{\prime}}=\frac{64 \pi^{4}}{3 h^{4} c^{3}} \frac{E_{v^{\prime}, J^{\prime}}-E_{v^{\prime \prime}, J^{\prime \prime}}}{2 J^{\prime}+1} S_{v^{\prime \prime}, J^{\prime \prime}}^{v^{\prime}, J^{\prime}}\left|D_{v^{\prime \prime}, J^{\prime \prime}}^{v^{\prime}, J^{\prime}}\right|^{2}
\end{aligned}
$$

In these formulae, $S_{v^{\prime \prime}, J^{\prime \prime}}^{v^{\prime}, J^{\prime}}$ is the Hönl-London factor resulting from the angular integrations $\left(S_{v^{\prime \prime}, J^{\prime \prime}}^{v^{\prime}, J^{\prime}}=J^{\prime \prime}\right.$ for $\mathrm{P}$ branches and $J^{\prime \prime}+1$ for R branches).

The radial transition matrix element $D_{v^{\prime \prime}, J^{\prime \prime}}^{v^{\prime}, J^{\prime}}$ is:

$$
D_{v^{\prime \prime}, J^{\prime \prime}}^{v^{\prime}, J^{\prime}}=\int_{0}^{\infty} \Psi_{v^{\prime \prime}, J^{\prime \prime}}(R) M(R) \Psi_{v^{\prime}, J^{\prime}}(R) \mathrm{d} R
$$

$M$ (in Debye) is the EDMF of the molecule in its ground electronic state $\mathrm{X}^{1} \Sigma^{+} .\left(1 \mathrm{D}=10^{-18} \mathrm{esu}, 1\right.$ $\mathrm{au}=2.541726645 \mathrm{D}$ and 1 C.m $\left.=2.9979245810^{11} \mathrm{esu}\right)$.

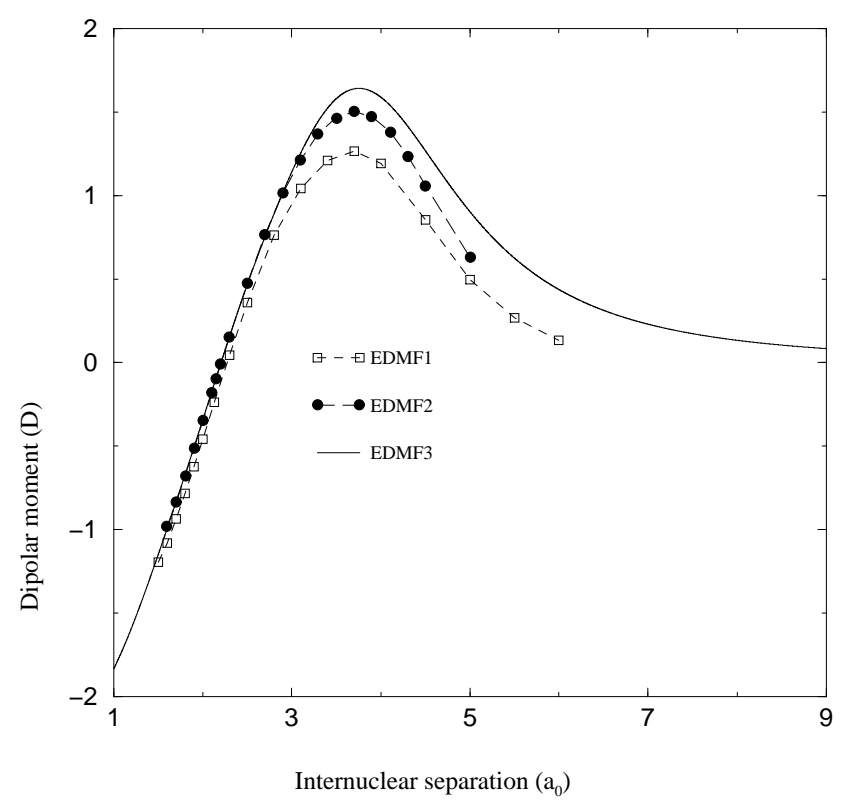

Fig. 1. The three EDMFs (in Debye) used in this work as a function of the internuclear distance (in $a_{0}$ ). EDMF1 = Werner (1981); EDMF2 = Langhoff \& Bauschlicher 1995; EDMF3 = Chackerian et al. 1984

\section{About the choice of the dipole moment function}

The theoretical determination of the permanent dipole moment of $\mathrm{CO}$ at equilibrium (when $R=R_{\mathrm{e}}$ and $R_{\mathrm{e}} \approx$ $2.132 a_{0}$ ) is a very difficult task since it involves cancellation of terms of about the same order of magnitude. Its experimental value is nowadays determined from Stark effect measurements on pure rotational transitions (Muenter $1975)$ and is equal to $-0.123 \mathrm{D}$. Rovibrational transition probabilities $(\Delta v \geq 1)$ on the other hand are most sensitive to the actual dependence of the EDMF with internuclear distance.

In a preliminary paper (Huré \& Roueff 1993), we have reported a set of $D$-values using the EDMF of Cooper \& Kirby (1987). This first investigation showed significant differences of the order of $10 \%$ with experimental determinations (Chackerian et al. 1984), even for low lying $v J$ states. We have repeated our calculations with two different theoretical ab-initio EDMFs. The first one, named EDMF1, has been computed by Werner (1981). The second one, named EDMF2, has been recently published by Langhoff \& Bauschlicher (1995). In addition, as we shall see below, EDMF1 and EDMF2 allow very satisfactory agreement with experimental results obtained at low excitations by Goorvitch \& Chackerian (1994a, b). We have indeed reproduced the values obtained by these authors by 
Table 3. $D$-values for some specific transitions. Values in the rectangles correspond to some transitions which are observed in the sun: $1-0$ to $20-19\left(J^{\prime} \leq 133\right) ; 2-0$ to $14-12\left(J^{\prime} \leq 110\right)$ for ${ }^{2} \mathrm{C}^{16} \mathrm{O}$ e.g. (Numbers in parenthesis refer to the power of ten involved)

\begin{tabular}{|c|c|c|c|c|c|c|c|}
\hline \multirow[b]{2}{*}{ Isotopes } & \multirow[b]{2}{*}{$\Delta v$} & \multirow[b]{2}{*}{$v^{\prime \prime}$} & \multirow[b]{2}{*}{$v^{\prime} \quad \Gamma$} & \multirow[b]{2}{*}{$E\left(\mathrm{~cm}^{-1}\right)$} & \multicolumn{3}{|c|}{$D$-values } \\
\hline & & & & & $D_{1}$ & $D_{2}$ & $D_{3}$ \\
\hline \multirow[t]{5}{*}{12,16} & 1 & $\begin{array}{rr}0 & 0 \\
40 & 133 \\
\end{array}$ & $\begin{array}{rr}1 & 0 \\
41 & 132 \\
\end{array}$ & $\begin{array}{r}2143.407 \\
464.961 \\
\end{array}$ & $\begin{array}{l}+1.069744(-1) \\
+3.888596(-1)\end{array}$ & $\begin{array}{l}+1.076565(-1) \\
+4.473379(-1) \\
\end{array}$ & $\begin{array}{l}+1.063450(-1) \\
+5.076834(-1)\end{array}$ \\
\hline & & $\begin{array}{rr}0 & 132 \\
2 & 133 \\
19 & 45 \\
\end{array}$ & $\begin{array}{rr}1 & 133 \\
3 & 132 \\
20 & 44 \\
\end{array}$ & $\begin{array}{l}2285.640 \\
1336.615 \\
1477.437\end{array}$ & $\begin{array}{l}+1.133904(-1) \\
+1.966144(-1) \\
+4.483567(-1)\end{array}$ & $\begin{array}{l}+1.122065(-1) \\
+2.007720(-1) \\
+4.640950(-1)\end{array}$ & $\begin{array}{l}+1.101410(-1) \\
+1.971536(-1) \\
+4.679886(-1)\end{array}$ \\
\hline & 2 & $\begin{array}{rr}0 & 0 \\
39 & 133 \\
\end{array}$ & $\begin{array}{rr}2 & 0 \\
41 & 132 \\
\end{array}$ & $\begin{array}{l}4260.279 \\
1199.611 \\
\end{array}$ & $\begin{array}{l}-6.340108(-3) \\
-2.753283(-1)\end{array}$ & $\begin{array}{l}-6.527777(-3) \\
-2.955704(-1) \\
\end{array}$ & $\begin{array}{l}-6.670675(-3) \\
-3.056673(-1)\end{array}$ \\
\hline & & $\begin{array}{rr}0 & 109 \\
12 \quad 44 \\
\end{array}$ & $\begin{array}{rr}2 & 110 \\
14 \quad 45 \\
\end{array}$ & $\begin{array}{l}4223.216 \\
3714.386 \\
\end{array}$ & $\begin{array}{l}-9.620556(-3) \\
-8.026599(-2)\end{array}$ & $\begin{array}{l}-9.607787(-3) \\
-7.617387(-2)\end{array}$ & $\begin{array}{l}-9.705405(-3) \\
-7.726195(-2)\end{array}$ \\
\hline & 3 & $\begin{array}{rr}0 & 0 \\
38 & 133\end{array}$ & $\begin{array}{rr}3 & 0 \\
41 & 132\end{array}$ & $\begin{array}{l}6350.647 \\
1960.119\end{array}$ & $\begin{array}{l}+3.609903(-4) \\
+1.257997(-1)\end{array}$ & $\begin{array}{l}+4.136643(-4) \\
+1.267045(-1)\end{array}$ & $\begin{array}{l}+4.273249(-4) \\
+1.219023(-1)\end{array}$ \\
\hline \multirow[t]{3}{*}{13,16} & 1 & $\begin{array}{rr}0 & 0 \\
40 & 133 \\
\end{array}$ & $\begin{array}{rr}1 & 0 \\
41 & 132 \\
\end{array}$ & $\begin{array}{r}2096.205 \\
496.790 \\
\end{array}$ & $\begin{array}{l}+1.057770(-1) \\
+4.132171(-1) \\
\end{array}$ & $\begin{array}{l}+1.064516(-1) \\
+4.720510(-1) \\
\end{array}$ & $\begin{array}{l}+1.051566(-1) \\
+5.295498(-1)\end{array}$ \\
\hline & & $\begin{array}{rr}0 & 102 \\
13 & 70 \\
\end{array}$ & $\begin{array}{rr}1 & 103 \\
14 & 69 \\
\end{array}$ & $\begin{array}{l}2275.338 \\
1473.339 \\
\end{array}$ & $\begin{array}{l}+1.106226(-1) \\
+3.894796(-1) \\
\end{array}$ & $\begin{array}{l}+1.099103(-1) \\
+3.988425(-1) \\
\end{array}$ & $\begin{array}{l}+1.081887(-1) \\
+3.963493(-1)\end{array}$ \\
\hline & $\begin{array}{l}2 \\
3\end{array}$ & $\begin{array}{rr}0 & 0 \\
39 & 133 \\
0 & 0 \\
38 & 133\end{array}$ & $\begin{array}{rr}2 & 0 \\
41 & 132 \\
3 & 0 \\
41 & 132\end{array}$ & $\begin{array}{l}4167.048 \\
1255.368 \\
6212.541 \\
2044.644\end{array}$ & $\begin{array}{l}-6.194922(-3) \\
-2.688827(-1) \\
+3.484349(-4) \\
+1.173594(-1)\end{array}$ & $\begin{array}{l}-6.379481(-3) \\
-2.866479(-1) \\
+3.994183(-4) \\
+1.169874(-1)\end{array}$ & $\begin{array}{l}-6.519202(-3) \\
-2.953231(-1) \\
+4.122071(-4) \\
+1.102474(-1)\end{array}$ \\
\hline \multirow[t]{3}{*}{12,18} & 1 & $\begin{array}{rr}0 & 0 \\
40 & 133 \\
\end{array}$ & $\begin{array}{rr}1 & 0 \\
41 & 132 \\
\end{array}$ & $\begin{array}{r}2092.253 \\
498.754 \\
\end{array}$ & $\begin{array}{l}+1.056761(-1) \\
+4.152606(-1) \\
\end{array}$ & $\begin{array}{l}+1.063502(-1) \\
+4.741270(-1)\end{array}$ & $\begin{array}{l}+1.050565(-1) \\
+5.314614(-1) \\
\end{array}$ \\
\hline & & $\begin{array}{ll}0 & 90 \\
7 & 45 \\
\end{array}$ & $\begin{array}{ll}1 & 91 \\
8 & 44 \\
\end{array}$ & $\begin{array}{l}2272.724 \\
1732.232 \\
\end{array}$ & $\begin{array}{l}+1.09834(-1) \\
+2.968596(-1)\end{array}$ & $\begin{array}{l}+1.093011(-1) \\
+3.007020(-1)\end{array}$ & $\begin{array}{l}+1.076784(-1) \\
+2.950279(-1)\end{array}$ \\
\hline & $\begin{array}{l}2 \\
3\end{array}$ & $\begin{array}{rr}0 & 2 \\
39 & 133 \\
0 & 0 \\
38 & 133\end{array}$ & $\begin{array}{rr}0 & 0 \\
41 & 132 \\
3 & 0 \\
41 & 132\end{array}$ & $\begin{array}{l}4159.241 \\
1259.908 \\
6200.974 \\
2050.719\end{array}$ & $\begin{array}{l}-6.182778(-3) \\
-2.683949(-1) \\
+3.473931(-4) \\
+1.167619(-1)\end{array}$ & $\begin{array}{l}-6.367074(-3) \\
-2.859682(-1) \\
+3.982350(-4) \\
+1.162949(-1)\end{array}$ & $\begin{array}{l}-6.506529(-3) \\
-2.980042(-1) \\
+4.109521(-4) \\
+1.094245(-1)\end{array}$ \\
\hline \multirow[t]{3}{*}{12,17} & 1 & $\begin{array}{rr}0 & 0 \\
40 & 133 \\
\end{array}$ & $\begin{array}{rr}1 & 0 \\
41 & 132 \\
\end{array}$ & $\begin{array}{r}2116.428 \\
484.209 \\
\end{array}$ & $\begin{array}{l}+1.062916(-1) \\
+4.030157(-1) \\
\end{array}$ & $\begin{array}{l}+1.069694(-1) \\
+4.617415(-1)\end{array}$ & $\begin{array}{l}+1.056673(-1) \\
+5.202969\langle-1\rangle\end{array}$ \\
\hline & & $\begin{array}{ll}0 & 51 \\
2 & 20 \\
\end{array}$ & $\begin{array}{ll}1 & 52 \\
3 & 19 \\
\end{array}$ & $\begin{array}{l}2261.660 \\
1984.913\end{array}$ & $\begin{array}{l}+1.083181(-1) \\
+1.834412(-1)\end{array}$ & $\begin{array}{l}+1.083328(-1) \\
+1.850181(-1) \\
\end{array}$ & $\begin{array}{l}+1.069200(-1) \\
+1.824928(-1)\end{array}$ \\
\hline & $\begin{array}{l}2 \\
3\end{array}$ & $\begin{array}{rr}0 & 0 \\
39 & 133 \\
0 & 0 \\
38 & 133\end{array}$ & $\begin{array}{rr}2 & 0 \\
41 & 132 \\
3 & 0 \\
41 & 132\end{array}$ & $\begin{array}{l}4206.996 \\
1231.835 \\
6271.722 \\
2010.439\end{array}$ & $\begin{array}{l}-6.257093(-3) \\
-2.707959(-1) \\
+3.537873(-4) \\
+1.207348(-1)\end{array}$ & $\begin{array}{l}-6.442993(-3) \\
-2.895178(-1) \\
+4.054966(-4) \\
+1.208871(-1)\end{array}$ & $\begin{array}{l}-6.584076(-3) \\
+5.378455(-1) \\
+4.186553(-4) \\
+1.149004(-1)\end{array}$ \\
\hline
\end{tabular}

using their analytical EDMF (see Chackerian et al. 1984), named EDMF3.

In a more recent paper (Roueff \& Huré 1995), we have compared $D$-values using EDMF1 to those obtained from EDMF3. For the rotationless $D$-values involving the lowest levels of to $\Delta v=1$ band, agreements are significantly better: less than $1 \%$ for $v$-numbers smaller than 10 . But differences of the order of $3 \%$ and $20 \%$ remain for the $\Delta v$ $=2$ and 3 bands respectively in the same conditions. It appears that EDMF1 leads to better results than the EDMF of Cooper \& Kirby (1987) does. However, EDMF2 might be preferable in some cases.

As shown in Table 1, EDMF2 and EDMF3 are identical at the equilibrium distance, but the first derivatives of the three EDMF at $R=R_{\mathrm{e}}$ differ by less than one percent. Figure 1 displays the 3 functions.

\section{Calculations and discussion}

The properties of the potential $V(R)$ as well as the integration method of the Schrödinger Eq. (1) have been described elsewhere (see Huré \& Roueff 1993). Since numerous energy levels are well known from experiments, we have been able to check the accuracy of our calculations of the energy levels by comparing them with the spectroscopic determinations (see Farrenq et al. 1991; Sauval et al. 1992; Le Floch 1991; Authier et al. 1993).

We have considered the most abundant isotopomers, namely: ${ }^{12} \mathrm{C}^{16} \mathrm{O},{ }^{13} \mathrm{C}^{16} \mathrm{O},{ }^{12} \mathrm{C}^{18} \mathrm{O}$ and ${ }^{12} \mathrm{C}^{17} \mathrm{O}$. The elec- 

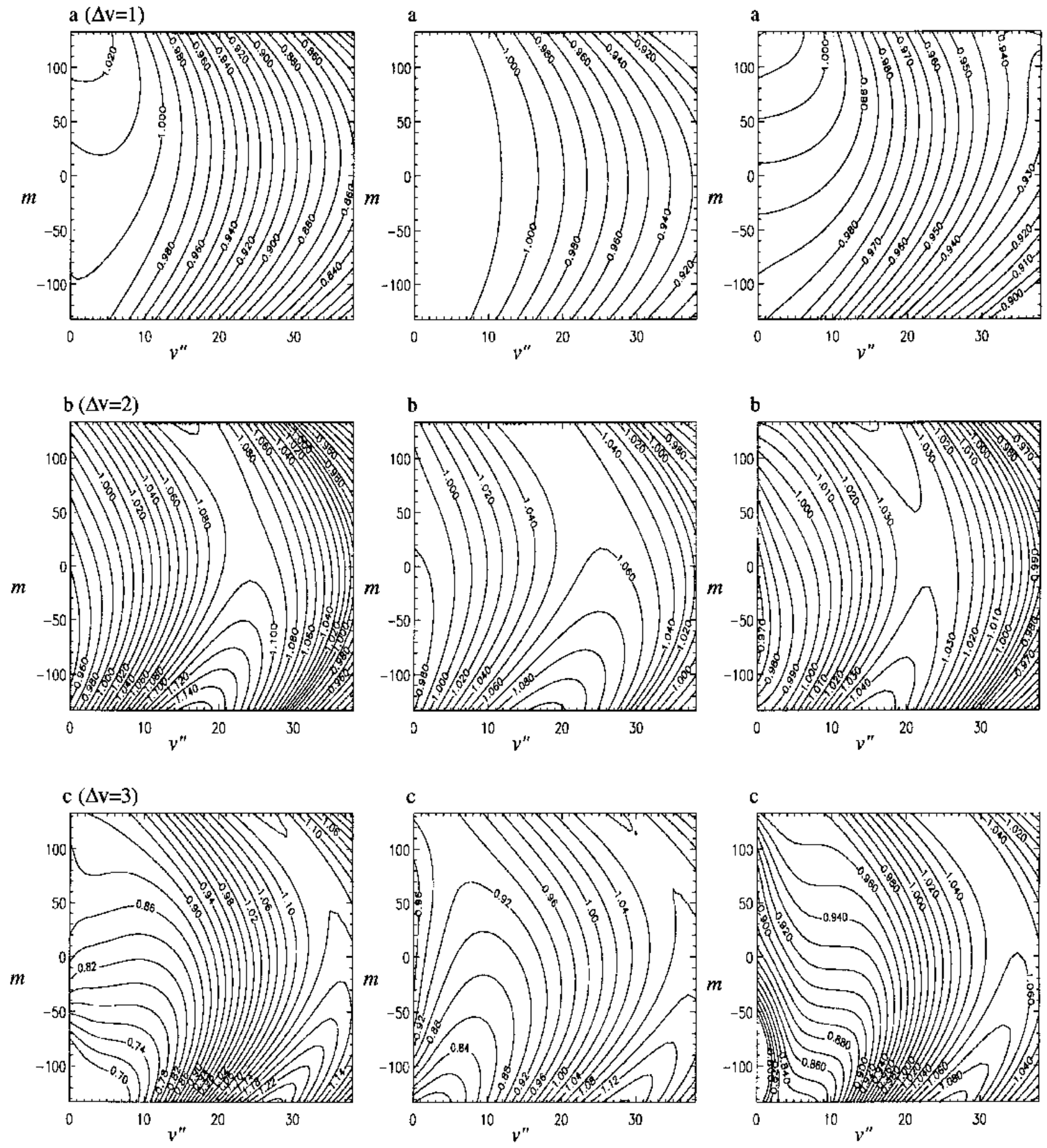

Figures 2

Figures 3

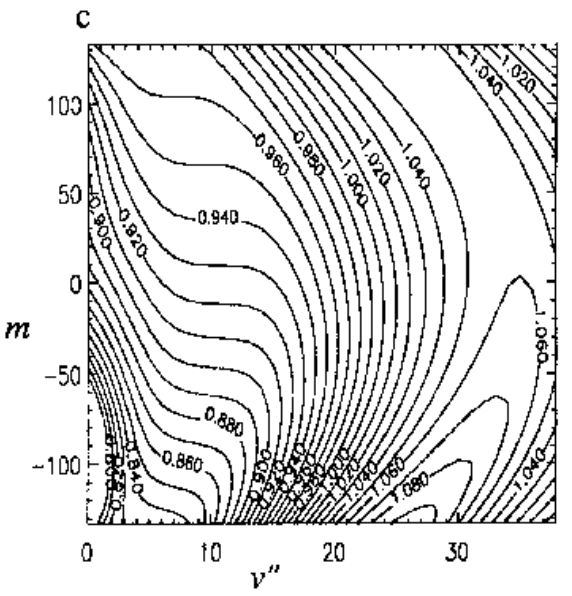

$\left(D_{1} / D_{3}\right)$

$\left(D_{2} / D_{3}\right)$

Figures 4

$\left(D_{1} / D_{2}\right)$

Fig. 2. to 4. Contour maps displaying the ratios of $D$-values as a function of $v^{\prime \prime}$ and $m$ for each band. $D_{1} / D_{3}$ are plotted in Fig. 2. $D_{2} / D_{3}$ are displayed in Fig. 3. $D_{1} / D_{2}$ are displayed in Figs. 4 . From top to bottom: $\Delta v=1$ (case a), $\Delta v=2$ (case b) and $\Delta v=3($ case $\mathrm{c})$ 
Table 4. a) Expansion coefficients of the polynomial expansion representing the transition $D$-values for the $\Delta v=1$ band and the ${ }^{12} \mathrm{C}^{16} \mathrm{O}$ isotope. Results are obtained using EDMF2 (Langhoff \& Bauschlicher 1995). The fits are better than 1\% when $|m| \leq m_{\max }$. The first column gives the lower vibrational quantum number $v^{\prime \prime}$. The headers come from formula 5 . The rotationless transition matrix element (in Debye) is displayed in the second column. Numbers in parenthesis refer to the power of ten involved

\begin{tabular}{|c|c|c|c|c|c|c|}
\hline$v^{n}$ & $\left|m_{\max }\right|$ & $\alpha_{0, v^{n}, 1}$ & $\alpha_{1, v^{\prime \prime}, 1}$ & $\alpha_{2, v^{\prime \prime}, 1}$ & $\alpha_{3, v^{n}, 1}$ & $\alpha_{4, v^{n}, 1}$ \\
\hline 00 & 133 & $+1.0765(-01)$ & $+1.14(-05)$ & $+3.85(-07)$ & $-1.39(-09)$ & $-1.48(-12)$ \\
\hline 01 & 133 & $+1.5212(-01)$ & $+1.23(-05)$ & $+5.27(-07)$ & $-2.02(-09)$ & $-2.34(-12)$ \\
\hline 02 & 133 & $+1.8610(-01)$ & $+1.03(-05)$ & $+6.25(-07)$ & $-2.53(-09)$ & $-3.23(-12)$ \\
\hline 03 & 133 & $+2.1463(-01)$ & $+6.44(-06)$ & $+6.93(-07)$ & $-3.00(-09)$ & $-4.03(-12)$ \\
\hline 04 & 133 & $+2.3959(-01)$ & $+8.96(-07)$ & $+7.50(-07)$ & $-3.44(-09)$ & $-5.17(-12)$ \\
\hline 05 & 133 & $+2.6201(-01)$ & $-5.91(-06)$ & $+7.86(-07)$ & $-3.86(-09)$ & $-6.11(-12)$ \\
\hline 06 & 133 & $+2.8244(-01)$ & $-1.39(-05)$ & $+8.08(-07)$ & $-4.27(-09)$ & $-6.98(-12)$ \\
\hline 07 & 133 & $+3.0127(-01)$ & $-2.31(-05)$ & $+8.23(-07)$ & $-4.68(-09)$ & $-8.19(-12)$ \\
\hline 08 & 133 & $+3.1874(-01)$ & $-3.33(-05)$ & $+8.30(-07)$ & $-5.08(-09)$ & $-9.54(-12)$ \\
\hline 09 & 133 & $+3.3506(-01)$ & $-4.44(-05)$ & $+8.26(-07)$ & $-5.48(-09)$ & $-1.09(-11)$ \\
\hline 10 & 133 & $+3.5035(-01)$ & $-5.65(-05)$ & $+8.14(-07)$ & $-5.89(-09)$ & $-1.23(-11)$ \\
\hline 11 & 133 & $+3.6471(-01)$ & $-6.95(-05)$ & $+7.94(-07)$ & $-6.30(-0.9)$ & $-1.39(-11)$ \\
\hline 12 & 133 & $+3.7823(-01)$ & $-8.33(-05)$ & $+7.67(-07)$ & $-6.71(-09)$ & $-1.57(-11)$ \\
\hline 13 & 133 & $+3.9098(-01)$ & $-9.80(-05)$ & $+7.33(-07)$ & $-7.12(-09)$ & $-1.76(-11)$ \\
\hline 14 & 133 & $+4.0299(-01)$ & $-1.14(-04)$ & $+6.91(-07)$ & $-7.54(-09)$ & $-1.97(-11)$ \\
\hline 15 & 133 & $+4.1432(-01)$ & $-1.30(-04)$ & $+6.41(-07)$ & $-7.96(-09)$ & $-2.19(-11)$ \\
\hline 16 & 133 & $+4.2499(-01)$ & $-1.47(-04)$ & $+5.83(-07)$ & $-8.39(-09)$ & $-2.44(-11)$ \\
\hline 17 & 133 & $+4.3503(-01)$ & $-1.65(-04)$ & $+5.17(-07)$ & $-8.82(-09)$ & $-2.70(-11)$ \\
\hline 18 & 133 & $+4.4446(-01)$ & $-1.84(-04)$ & $+4.44(-07)$ & $-9.26(-09)$ & $-3.00(-11)$ \\
\hline 19 & 133 & $+4.5330(-01)$ & $-2.03(-04)$ & $+3.62(-07)$ & $-9.70(-09)$ & $-3.31(-11)$ \\
\hline 20 & 133 & $+4.6155(-01)$ & $-2.23(-04)$ & $+2.72(-07)$ & $-1.02(-08)$ & $-3.66(-11)$ \\
\hline 21 & 133 & $+4.6922(-01)$ & $-2.44(-04)$ & $+1.73(-07)$ & $-1.06(-08)$ & $-4.04(-11)$ \\
\hline 22 & 133 & $+4.7632(-01)$ & $-2.66(-04)$ & $+6.48(-08)$ & $-1.11(-08)$ & $-4.46(-11)$ \\
\hline 23 & 133 & $+4.8285(-01)$ & $-2.88(-04)$ & $-5.30(-08)$ & $-1.15(-08)$ & $-4.92(-11)$ \\
\hline 24 & 133 & $+4.8881(-01)$ & $-3.11(-04)$ & $-1.81(-07)$ & $-1.20(-08)$ & $-5.42(-11)$ \\
\hline 25 & 133 & $+4.9419(-01)$ & $-3.35(-04)$ & $-3.21(-07)$ & $-1.25(-08)$ & $-5.97(-11)$ \\
\hline 26 & 133 & $+4.9899(-01)$ & $-3.59(-04)$ & $-4.72(-07)$ & $-1.29(-08)$ & $-6.58(-11)$ \\
\hline 27 & 133 & $+5.0319(-01)$ & $-3.84(-04)$ & $-6.21(-07)$ & $-1.34(-08)$ & $-7.35(-11)$ \\
\hline 28 & 133 & $+5.0663(-01)$ & $-4.09(-04)$ & $-6.91(-07)$ & $-1.40(-08)$ & $-8.83(-11)$ \\
\hline 29 & 133 & $+5.0891(-01)$ & $-4.32(-04)$ & $-4.73(-07)$ & $-1.49(-08)$ & $-1.25(-10)$ \\
\hline 30 & 133 & $+5.1008(-01)$ & $-4.51(-04)$ & $+3.90(-08)$ & $-1.64(-08)$ & $-1.86(-10)$ \\
\hline 31 & 133 & $+5.1146(-01)$ & $-4.70(-04)$ & $+1.48(-07)$ & $-1.86(-08)$ & $-2.31(-10)$ \\
\hline 32 & 133 & $+5.1330(-01)$ & $-4.96(-04)$ & $-3.73(-07)$ & $-2.00(-08)$ & $-2.41(-10)$ \\
\hline 33 & 133 & $+5.1450(-01)$ & $-5.19(-04)$ & $-1.08(-06)$ & $-2.19(-08)$ & $-2.45(-10)$ \\
\hline 34 & 133 & $+5.1552(-01)$ & $-5.30(-04)$ & $-2.38(-06)$ & $-2.48(-08)$ & $-2.10(-10)$ \\
\hline 35. & 133 & $+5: 1604(-01)$ & $-5.84(-04)$ & $-3.64(-06)$ & $-2.36(-08)$ & $-1.88(-10)$ \\
\hline 36 & 133 & $+5.1244(-01)$ & $-6.52(-04)$ & $-4.01(-06)$ & $-2.18(-08)$ & $-2.13(-10)$ \\
\hline 37 & 133 & $+5.0788(-01)$ & $-6.95(-04)$ & $-4.63(-06)$ & $-2.19(-08)$ & $-2.23(-10)$ \\
\hline 38 & 129 & $+5.0196(-01)$ & $-7.40(-04)$ & $-5.25(-06)$ & $-2.15(-08)$ & $-2.30(-10)$ \\
\hline 39 & 131 & $+4.9378(-01)$ & $-7.79(-04)$ & $-5.28(-06)$ & $-2.18(-08)$ & $-2.80(-10)$ \\
\hline 40 & 130 & $+4.8536(-01)$ & $-8.15(-04)$ & $-5.69(-06)$ & $-2.22(-08)$ & $-3.09(-10)$ \\
\hline
\end{tabular}

Table $4 \mathrm{a}-\Delta v=1$ for ${ }^{12} \mathrm{C}^{16} \mathrm{O}$.

tronic properties such as the interatomic potential and the EDMF are independent of the isotope. However the rovibrational energy levels and the corresponding wavefunctions, which are obtained from the solution of the Schrödinger equation, depend on the reduced mass of the system. Table 2 summarizes the values of the reduced mass for the various isotopomers.

We have computed $D$-values for transitions involving $v^{\prime} \leq 41$ and $J^{\prime} \leq 133$ for $\Delta v=1,2$ and 3 using EDMF 1 , EDMF2 and EDMF3 (corresponding $D$-values are noted $D_{1}, D_{2}$ and $D_{3}$ respectively). A simple cubic spline has been applied to interpolate the ab-initio data points (except for EDMF3 which is analytical). The adopted limits on $v$ and $J$ correspond to the highest (independently) observed levels for ${ }^{12} \mathrm{C}^{16} \mathrm{O}$ molecule in the solar spectrum detected by the ATMOS experiment (Farmer \& Norton 1989; Farrenq et al. 1991).

We point out that the computation of the $D$-values seems to be very sensitive to the interpolation method used to calculate the electric dipole moment functions between data points. Actually, severe differences appear on $D$-values when using a Padé approximant instead of a cubic spline procedure.

We display in Figs. 2 to 4 the contour plots of the ratios of the $D$-values calculated with the three EDMF's for the $\Delta v=1,2$ and 3 bands (a, b and c respectively) 
Table 4. b) Same as Table 4 a for $\Delta v=2$

\begin{tabular}{|c|c|c|c|c|c|c|}
\hline$v^{\prime \prime}$ & $\left|m_{\max }\right|$ & $\alpha_{0, v^{*}, 2}$ & $\alpha_{1, v^{*}, 2}$ & $\alpha_{2, v^{\pi}, 2}$ & $\alpha_{3, v^{*}, 2}$ & $\alpha_{4, v^{*}, 2}$ \\
\hline 00 & 133 & $-6.5274(-03)$ & $-1.68(-05)$ & $-9.23(-08)$ & $-2.67(-11)$ & $-5.42(-13)$ \\
\hline 01 & 133 & $-1.1458(-02)$ & $-2.89(-05)$ & $-1.57(-07)$ & $-4.02(-11)$ & $-1.05(-12)$ \\
\hline 02 & 133 & $-1.6391(-02)$ & $-4.06(-05)$ & $-2.25(-07)$ & $-4.50(-11)$ & $-1.36(-12)$ \\
\hline 03 & 133 & $-2.1418(-02)$ & $-5.20(-05)$ & $-2.88(-07)$ & $-4.36(-11)$ & $-1.86(-12)$ \\
\hline 04 & 133 & $-2.6540(-02)$ & $-6.31(-05)$ & $-3.52(-07)$ & $-3.81(-11)$ & $-2.30(-12)$ \\
\hline 05 & 133 & $-3.1760(-02)$ & $-7.41(-05)$ & $-4.18(-07)$ & $-1.68(-11)$ & $-2.59(-12)$ \\
\hline 06 & 133 & $-3.7088(-02)$ & $-8.47(-05)$ & $-4.83(-07)$ & $+5.89(-12)$ & $-2.87(-12)$ \\
\hline 07 & 133 & $-4.2514(-02)$ & $-9.51(-05)$ & $-5.45(-07)$ & $+3.72(-11)$ & $-3.29(-12)$ \\
\hline 08 & 133 & $-4.8036(-02)$ & $-1.05(-04)$ & $-6.09(-07)$ & $+8.10(-11)$ & $-3.59(-12)$ \\
\hline 09 & 133 & $-5.3649(-02)$ & $-1.15(-04)$ & $-6.73(-07)$ & $+1.34(-10)$ & $-3.80(-12)$ \\
\hline 10 & 133 & $-5.9356(-02)$ & $-1.25(-04)$ & $-7.35(-07)$ & $+1.97(-10)$ & $-4.04(-12)$ \\
\hline 11 & 133 & $-6.5151(-02)$ & $-1.34(-04)$ & $-7.98(-07)$ & $+2.69(-10)$ & $-4.27(-12)$ \\
\hline 12 & 133 & $-7.1032(-02)$ & $-1.43(-04)$ & $-8.59(-07)$ & $+3.57(-10)$ & $-4.46(-12)$ \\
\hline 13 & 133 & $-7.6995(-02)$ & $-1.52(-04)$ & $-9.20(-07)$ & $+4.57(-10)$ & $-4.64(-12)$ \\
\hline 14 & 133 & $-8.3036(-02)$ & $-1.60(-04)$ & $-9.80(-07)$ & $+5.69(-10)$ & $-4.81(-12)$ \\
\hline 15 & 133 & $-8.9156(-02)$ & $-1.68(-04)$ & $-1.04(-06)$ & $+6.95(-10)$ & $-4.93(-12)$ \\
\hline 16 & 133 & $-9.5350(-02)$ & $-1.76(-04)$ & $-1.10(-06)$ & $+8.38(-10)$ & $-5.09(-12)$ \\
\hline 17 & 133 & $-1.0161(-01)$ & $-1.83(-04)$ & $-1.15(-06)$ & $+9.97(-10)$ & $-5.19(-12)$ \\
\hline 18 & 133 & $-1.0794(-01)$ & $-1.90(-04)$ & $-1.21(-06)$ & $+1.17(-09)$ & $-5.31(-12)$ \\
\hline 19 & 133 & $-1.1434(-01)$ & $-1.96(-04)$ & $-1.26(-06)$ & $+1.37(-09)$ & $-5.44(-12)$ \\
\hline 20 & 133 & $-1.2079(-01)$ & $-2.02(-04)$ & $-1.31(-06)$ & $+1.59(-09)$ & $-5.52(-12)$ \\
\hline 21 & 133 & $-1.2731(-01)$ & $-2.07(-04)$ & $-1.36(-06)$ & $+1.82(-09)$ & $-5.61(-12)$ \\
\hline 22 & 133 & $-1.3387(-01)$ & $-2.12(-04)$ & $-1.41(-06)$ & $+2.09(-09)$ & $-5.64(-12)$ \\
\hline 23 & 133 & $-1.4048(-01)$ & $-2.17(-04)$ & $-1.46(-06)$ & $+2.38(-09)$ & $-5.64(-12)$ \\
\hline 24 & 133 & $-1.4714(-01)$ & $-2.21(-04)$ & $-1.51(-06)$ & $+2.70(-09)$ & $-5.56(-12)$ \\
\hline 25 & 133 & $-1.5384(-01)$ & $-2.24(-04)$ & $-1.56(-06)$ & $+3.05(-09)$ & $-5.33(-12)$ \\
\hline 26 & 133 & $-1.6057(-01)$ & $-2.27(-04)$ & $-1.61(-06)$ & $+3.44(-09)$ & $-4.75(-12)$ \\
\hline 27 & 133 & $-1.6734(-01)$ & $-2.30(-04)$ & $-1.65(-06)$ & $+3.93(-09)$ & $-4.35(-12)$ \\
\hline 28 & 133 & $-1.7438(-01)$ & $-2.35(-04)$ & $-1.57(-06)$ & $+4.63(-09)$ & $-1.16(-11)$ \\
\hline 29 & 133 & $-1.8205(-01)$ & $-2.37(-04)$ & $-1.16(-06)$ & $+5.38(-09)$ & $-4.14(-11)$ \\
\hline 30 & 131 & $-1.8962(-01)$ & $-2.38(-04)$ & $-7.23(-07)$ & $+6.07(-09)$ & $-8.01(-11)$ \\
\hline 31 & 133 & $-1.9652(-01)$ & $-2.46(-04)$ & $-6.65(-07)$ & $+8.16(-09)$ & $-9.41(-11)$ \\
\hline 32 & 127 & $-2.0251(-01)$ & $-2.70(-04)$ & $-1.31(-06)$ & $+1.08(-08)$ & $-7.01(-11)$ \\
\hline 33 & 120 & $-2.0753(-01)$ & $-2.57(-04)$ & $-1.99(-06)$ & $+1.11(-08)$ & $-4.35(-11)$ \\
\hline 34 & 113 & $-2.1360(-01)$ & $-2.39(-04)$ & $-2.64(-06)$ & $+1.11(-08)$ & $-8.01(-12)$ \\
\hline 35 & 124 & $-2.2027(-01)$ & $-2.23(-04)$ & $-3.42(-06)$ & $+1.12(-08)$ & $+5.65(-11)$ \\
\hline 36 & 133 & $-2.2963(-01)$ & $-2.19(-04)$ & $-3.28(-06)$ & $+1.32(-08)$ & $+6.61(-11)$ \\
\hline 37 & 133 & $-2.3914(-01)$ & $-2.07(-04)$ & $-3.07(-06)$ & $+1.43(-08)$ & $+7.38(-11)$ \\
\hline 38 & 133 & $-2.4876(-01)$ & $-2.02(-04)$ & $-2.70(-06)$ & $+1.62(-08)$ & $+6.92(-11)$ \\
\hline 39 & 133 & $-2.5621(-01)$ & $-1.84(-04)$ & $-2.69(-06)$ & $+1.72(-08)$ & $+8.54(-11)$ \\
\hline
\end{tabular}

Table $4 \mathrm{~b}-\Delta v=2$ for ${ }^{12} \mathrm{C}^{16} \mathrm{O}$.

and the complete set of $v^{\prime \prime}$ and $m$ values. The ratios are: $D_{1} / D_{3}$ in Fig. 2, $D_{2} / D_{3}$ in Fig. 3 and $D_{1} / D_{2}$ in Figs. 4. These plots concern the ${ }^{12} \mathrm{C}^{16} \mathrm{O}$ molecule.

For low $v$ and $J$ transitions inside the fundamental band of the ${ }^{12} \mathrm{C}^{16} \mathrm{O}$ molecule, the three EDMF lead to very similar results. Agreement is good to within less than $1 \%$. EDMF1 leads to $D$-values slightly closer to the experimental results than EDMF2 according to the first derivatives at $R=R_{\mathrm{e}}$. For extreme $v$ and $J$ transitions, differences appear as large as $20 \%$, especially when using EDMF1 as shown in Roueff \& Huré (1995). For intermediate $v$ and $J$ values, agreement between the EDMF's is quite good within a few percent. This is the case for most of the lines recently identified in the solar spectrum (see Table 3). For the first overtone band $(\Delta v=2)$, we see that EDMF1 and EDMF2 lead to very close values of dipole matrix elements for the whole set of $v J$-lines within $2-3 \%$. Comparing with EDMF3, the shift is largest for intermediate $v$-numbers $(v \approx 20)$ and largest $J$-numbers. Finally, for the second overtone band $(\Delta v=3)$, the three EDMF lead to very different $D$-values. As shown from Eq. (2), larger differences do exist between $g f$-values based on the three different EDMF.

The differences obtained from the three EDMFs remain of the same order of magnitude for the ${ }^{13} \mathrm{C}^{16} \mathrm{O}$ molecule and other isotopomers, which reflects the coherence of the EDMF over all involved internuclear distances. This is not the case when two experimental EDMFs (Chackerian \& Tipping 1983 and Chackerian et al. 1984) are compared as shows Goorvitch (1994) in his Table 5.

Globally, whereas the ratios $D_{1} / D_{3}, D_{2} / D_{3}$ and $D_{1} / D_{2}$ remain close to 1 for most values of $v^{\prime \prime}$ and $m$ of the $\Delta v=1$ band, we note that the discrepancies between both choices of EDMF increase as $\Delta v, v^{\prime}$ and $m$ increase. We feel that these results show the remaining uncertainties in the $D$-values due to the different choices 
Table 4. c) Same as Table 4 a for $\Delta v=3$

\begin{tabular}{|c|c|c|c|c|c|c|}
\hline$v^{\prime \prime}$ & $\left|m_{\max }\right|$ & $\alpha_{0, v^{*}, 3}$ & $\alpha_{1, v^{\prime \prime}, 3}$ & $\alpha_{2, v^{\mu}, 3}$ & $\alpha_{3, v^{n}, 1}$ & $\alpha_{4, v^{\prime \prime}, 3}$ \\
\hline$\overline{00}$ & 133 & $+4.1405(-04)$ & $+2.44(-06)$ & $+1.27(-08)$ & $+2.80(-11)$ & $+2.12(-13)$ \\
\hline 01 & 133 & $+8.5412(-04)$ & $+4.91(-06)$ & $+2.67(-08)$ & $+5.47(-11)$ & $+3.58(-13)$ \\
\hline 02 & 133 & $+1.3896(-03)$ & $+7.78(-06)$ & $+4.40\langle-08\}$ & $+8.62(-11)$ & $+4.99(-13)$ \\
\hline 03 & 133 & $+2.0375(-03)$ & $+1.10(-05)$ & $+5.90(-08)$ & $+1.21(-10)$ & $+9.13(-13)$ \\
\hline 04 & 133 & $+2.7736(-03)$ & $+1.46(-05)$ & $+7.91(-08)$ & $+1.59(-10)$ & $+1.23(-12)$ \\
\hline 05 & 133 & $+3.6091(-03)$ & $+1.85(-05)$ & $+1.02(-07)$ & $+2.02(-10)$ & $+1.51(-12)$ \\
\hline 06 & 133 & $+4.5438(-03)$ & $+2.27(-0.5)$ & $+1.27(-07)$ & $+2.42(-10)$ & $+1.86(-12)$ \\
\hline 07 & 133 & $+5.5794(-03)$ & $+2.72(-05)$ & $+1.54(-07)$ & $+2.86(-10)$ & $+2.23(-12)$ \\
\hline 08 & 133 & $+6.7167(-03)$ & $+3.19(-05)$ & $+1.83(-07)$ & $+3.31(-10)$ & $+2.59(-12)$ \\
\hline 09 & 133 & $+7.9571(-03)$ & $+3.69(-05)$ & $+2.15(-07)$ & $+3.76(-10)$ & $+2.96(-12)$ \\
\hline 10 & 133 & $+9.3010(-03)$ & $+4.21(-05)$ & $+2.49(-07)$ & $+4.21(-10)$ & $+3.34(-12)$ \\
\hline 11 & 133 & $+1.0750(-02)$ & $+4.75(-05)$ & $+2.85(-07)$ & $+4.66(-10)$ & $+3.73(-12)$ \\
\hline 12 & 133 & $+1.2308(-02)$ & $+5.31(-05)$ & $+3.22(-07)$ & $+5.11(-10)$ & $+4.15(-12)$ \\
\hline 13 & 133 & $+1.3975(-02)$ & $+5.89(-05)$ & $+3.61(-07)$ & $+5.54(-10)$ & $+4.58(-12)$ \\
\hline 14 & 133 & $+1.5752(-02)$ & $+6.49(-05)$ & $+4.02(-07)$ & $+5.95(-10)$ & $+5.03(-12)$ \\
\hline 15 & 133 & $+1.7642(-02)$ & $+7.10(-05)$ & $+4.44(-07)$ & $+6.34(-10)$ & $+5.49(-12)$ \\
\hline 16 & 133 & $+1.9645(-02)$ & $+7.73(-05)$ & $+4.88(-07)$ & $+6.70(-10)$ & $+5.98(-12)$ \\
\hline 17 & 133 & $+2.1762(-02)$ & $+8.37(-05)$ & $+5.33(-07)$ & $+7.04(-10)$ & $+6.48(-12)$ \\
\hline 18 & 133 & $+2.3993(-02)$ & $+9.02(-05)$ & $+5.79(-07)$ & $+7.33(-10)$ & $+6.99(-12)$ \\
\hline 19 & 133 & $+2.6340(-02)$ & $+9.69(-05)$ & $+6.27(-07)$ & $+7.58(-10)$ & $+7.51(-12)$ \\
\hline 20 & 133 & $+2.8802(-02)$ & $+1.04(-04)$ & $+6.76(-07)$ & $+7.78(-10)$ & $+8.03(-12)$ \\
\hline 21 & 133 & $+3.1381(-02)$ & $+1.10(-04)$ & $+7.27(-07)$ & $+7.93(-10)$ & $+8.55(-12)$ \\
\hline 22 & 133 & $+3.4075(-02)$ & $+1.17(-04)$ & $+7.79(-07)$ & $+8.03(-10)$ & $+9.08(-12)$ \\
\hline 23 & 133 & $+3.6887(-02)$ & $+1.24(-04)$ & $+8.31(-07)$ & $+8.06(-10)$ & $+9.71(-12)$ \\
\hline 24 & 133 & $+3.9816(-02)$ & $+1.31(-04)$ & $+8.83(-07)$ & $+8.01(-10)$ & $+1.04(-11)$ \\
\hline 25 & 133 & $+4.2860(-02)$ & $+1.38(-04)$ & $+9.36(-07)$ & $+7.84(-10)$ & $+1.11(-11)$ \\
\hline 26 & 133 & $+4.6026(-02)$ & $+1.45(-04)$ & $+9.86(-07)$ & $+7.43(-10)$ & $+1.21(-11)$ \\
\hline 27 & 133 & $+4.9375(-02)$ & $+1.52(-04)$ & $+1.00(-05)$ & $+6.75(-10)$ & $+1.57(-11)$ \\
\hline 28 & 133 & $+5.3092(-02)$ & $+1.60(-04)$ & $+8.70(-07)$ & $+5.93(-10)$ & $+2.96(-11)$ \\
\hline 29 & 126 & $+5.6617(-02)$ & $+1.66(-04)$ & $+8.79(-07)$ & $+5,47(-10)$ & $+3.68(-11)$ \\
\hline 30 & 118 & $+6.0195(-02)$ & $+1.73(-04)$ & $+9.13(-07)$ & $+4.99(-10)$ & $+4.45(-11)$ \\
\hline 31 & 111 & $+6.3957(-02)$ & $+1.79(-04)$ & $+8.77(-07)$ & $+4.46(-10)$ & $+6.26(-11)$ \\
\hline 32 & 105 & $+6.7844(-02)$ & $+1.85(-04)$ & $+7.84(-07)$ & $+4.16(-10)$ & $+9.61(-11)$ \\
\hline 33 & 110 & $+7.1774(-02)$ & $+1.86(-04)$ & $+7.90(-07)$ & $+1.22(-09)$ & $+1.35(-10)$ \\
\hline 34 & 101 & $+7.4757(-02)$ & $+1.92(-04)$ & $+1.50(-06)$ & $+1.28(-09)$ & $+1.20(-10)$ \\
\hline 35 & 92 & $+7.8791(-02)$ & $+2.01(-04)$ & $+2.29(-06)$ & $+1.13(-09)$ & $+8.43(-11)$ \\
\hline 36 & 105 & $+8.3444(-02)$ & $+2.13(-04)$ & $+3.64(-06)$ & $-5.61(-10)$ & $-6.09(-11)$ \\
\hline 37 & 110 & $+9.1414(-02)$ & $+2.24(-04)$ & $+3.21(-06)$ & $-1.58(-09)$ & $-3.38(-11)$ \\
\hline 38 & 133 & $+9.9492(-02)$ & $+2.34(-04)$ & $+2.58(-06)$ & $-3.34(-09)$ & $+1.28(-11)$ \\
\hline
\end{tabular}

Table $4 \mathrm{c}-\Delta v=3$ for ${ }^{12} \mathrm{C}^{16} \mathrm{O}$.

of the EDMF functions. Both $\Delta v=1$ and 2 transitions are present in the infrared solar spectrum (Grevesse \& Sauval 1991, 1993) and are often used to determine the temperature of the photosphere. In the particular case of $\Delta v=2$, we suggest that models of the photosphere should be performed with both sets of $D$-values to evaluate the uncertainties coming from the molecular data themselves.

\section{Polynomial fits}

For an intensive use of this set of numerous lines and associated $D$-values, it is very useful to have an analytical formula. Consequently, we have fitted them with a fourth order polynomial form for each sequence and each vibrational band by using a numerical minimax procedure from the NAG mathematical library as a function of $m=$ $\left[J^{\prime}\left(J^{\prime}+1\right)-J^{\prime \prime}\left(J^{\prime \prime}+1\right)\right] / 2, v^{\prime \prime}$ and $\Delta v=n$ :

$D_{n}^{v^{\prime \prime}}(m)=\left\langle v^{\prime \prime}+n, J^{\prime}|M| v^{\prime \prime}, J^{\prime \prime}\right\rangle=\alpha_{0, v^{\prime \prime}, n}+\alpha_{1, v^{\prime \prime}, n} m+$ $\alpha_{2, v^{\prime \prime}, n} m^{2}+\alpha_{3, v^{\prime \prime}, n} m^{3}+\alpha_{4, v^{\prime \prime}, n} m^{4}$

For the P-branch $(\Delta J=-1), m$ is $\left(-J^{\prime \prime}\right)$ and for the R-branch $(\Delta J=+1), m$ is $\left(+J^{\prime}\right)$.

This procedure is sufficient to reproduce most of the original values with a relative uncertainty less than $1 \%$. The accuracy depends on the values of $m$ and $v^{\prime \prime}$ involved. Figure 5 displays the results of the calculations for $v^{\prime \prime}=38$ and $\Delta v=1,2$ and 3 obtained with EDMF2 together with our obtained analytical fit. The relative error arising from the fitting procedure in logarithmic scale is also shown. Tables 4a-c give the polynomial expansion coefficients for the $\Delta v=1,2$ and 3 sequences for ${ }^{12} \mathrm{C}^{16} \mathrm{O}$ corresponding to EDMF2 which gives the best agreement with the experiments. The range of values of $m$ for which the error due to the fitting procedure is less than $1 \%$ is also listed (for example, we note that, for the fundamental $(\Delta v=1)$ band of the ${ }^{12} \mathrm{C}^{16} \mathrm{O}$ molecule, this error is less than $10^{-2}$ for $v^{\prime \prime}=38$ and $|m|$ smaller than 129). We feel that this ac- 

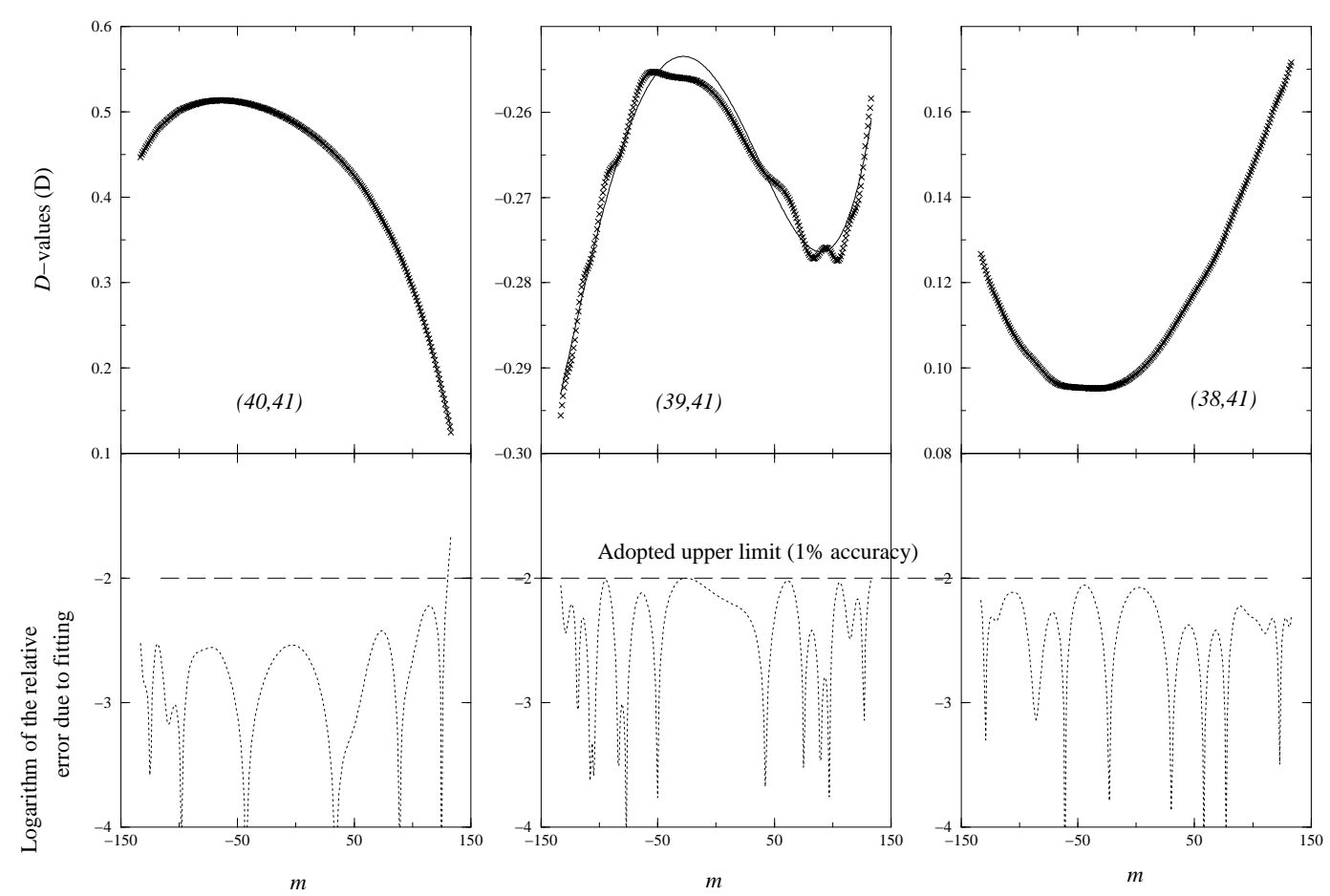

Fig. 5. $D$-values as a function of $m$ for the highest vibrational sequences considered (top). Crosses are the data. Lines are the polynomial fittings. Logarithm of the relative errors due to the fitting procedure is also shown (dotted, bottom plots)

curacy is sufficient for most applications and still smaller than the uncertainty due to the choice of the EDMF as we point out later.

Though the square of the transition matrix elements is involved in $f$-values or A Einstein coefficients, we found that the errors arising from the fitting of the $D$-values squared were always larger than two times the error coming from the fitting of the $D$-values themselves for the whole set of data. We found that the isotopic substitution has only a small effect on $D$-values. Consequently, we have obtained very close polynomial forms for the different isotopomers. Tables $4 \mathrm{a}-\mathrm{c}$ and similar tables for the ${ }^{13} \mathrm{C}^{16} \mathrm{O}$, ${ }^{12} \mathrm{C}^{18} \mathrm{O}$ and ${ }^{12} \mathrm{C}^{17} \mathrm{O}$ isotopomers are avalaible in electronic form at the CDS via anonymous ftp 130.79.128.5. The polynomial fits of $D_{1}$-values (computed with EDMF1) can also be obtained via e-mail on request to the authors.

Acknowledgements. We are grateful to A.J. Sauval and R. Bloome for their suggestions and comments. Most calculations have been performed on the CONVEX computer of ParisMeudon Observatory. We acknowledge support from the GDR 0127 of CNRS "Physico-Chimie des Molécules et des Grains Interstellaires" and from the "Société de Secours des Amis de la Science" at Paris.

\section{References}

Authier N., Bagland N., Le Floch A., 1993, J. Mol. Spectrosc. 160,590

Chackerian C. Jr., Tipping R.H., 1983, J. Mol. Spectrosc. 99, 431
Chackerian C. Jr., Farrenq R., Guelachvili G., C. Rossetti, Urban W., 1984, Can. J. Phys. 62, 1579

Chackerian C. Jr., Goorvitch D., Huré J.M., Roueff E., 1994, J. Mol. Spectrosc. 165, 583

Cooper D.L., Kirby K, 1987, J. Chem. Phys. 87, 424

Farrenq R., Guelachvili G., Sauval A.J., Grevesse N., Farmer C.B., 1991, J. Mol. Spectrosc. 149, 375

Framer C.B., Norton B., 1989, "A high-resolution atlas of the infrared spectrum of the sun and the earth atmosphere from space", NASA Ref. Publ. 1224, Vols. I and II

Goorvitch D., Chackerian C. Jr., 1994a, ApJS 91, 483

Goorvitch D., Chackerian C. Jr., 1994b, ApJS 92, 311

Goorvitch D., 1994, ApJS 95, 535

Grevesse N., Sauval A.J., 1991 , "Infrared Spectral Region of Stars". In: Jashek C. \& Andrillat Y. (eds.). Cambridge University Press, 215

Grevesse N., Sauval A.J., 1993, "Molecular Opacities in the Stellar Environment". In: Jorgensen U.G. (ed.). Springer Verlag, 196

Langhoff S.R., Bauschlicher C.W. 1995, J. Chem. Phys. 102, 5220

Huré J.M., Roueff E., 1993, J. Mol. Spectrosc. 160, 335

Roueff E., Huré J.M. 1995, in "Laboratory and Astronomical High Resolution Spectra", Astron. Soc. Pac. Conf. Ser. 81. In: Sauval, Blomme and Grevesse (eds.), 288

Le Floch A., 1991, A\&AS 90, 513

Muenter J.S., 1975, J. Mol. Spectrosc. 55, 490

Sauval A.J., Farrenq R., Guelachvili G., Grevesse N., Farmer C.B., Norton R.H., 1992, Astron. ans Astrophys. 265, 355

Steinfeld J.I., 1989, in "Molecules and Radiation", MIT Press Werner J., 1981, Mol. Phys. 44, 111 